\title{
OPTIMAL DESIGN FOR THE CASTING PROCESS OF THE LOWER CASE OF GEARBOX
}

\author{
Xin-yu QIN, Yong SU*, Rui-xiang ZHU,He-fa CHENG and Lan-jun LIU \\ School of materials science and Engineering, Hefei University of Technology, Hefei, Anhui, China \\ ${ }^{*}$ Corresponding author
}

Keywords: gearbox; nodular cast iron; numerical simulation

Abstract: The lower case is part of a gearbox to support the hub or bearing. The material of this casting is ductile iron. Process design is based on the paste-like solidification of ductile iron and the self-expanding characteristics of graphite[1]. The pouring system is designed according to the theory of large hole outflow. The cavity is filled with liquid metal fleetly and reposefully. Ultimately, the acquired casting has compact structure and no obvious shrinkage. By way of sand casting, furan resin sand is used as sand - type material. Throughout the design process, software such as UG、 VE、PROcast and CAD are used to support simulation. The parameters of casting process have been optimized to get better results.

\section{Introduction}

The lower case is a part of gearbox which is used in machines such as cars 、 boats and compressors (Fig. 1). Bearing socket and gear fixed socket can be found on the picture. Inside the lower case, there is a large cavity which is used to install and locate grooves and pits. On both sides of the box, stiffeners are provided to enhance the stability of structures.

\section{Material and properties of the casting}

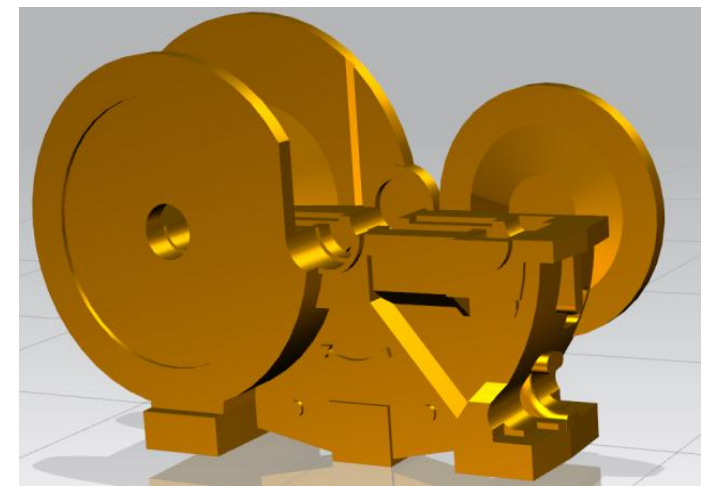

Fig. 1 shape of the lower house

The material is ductile iron and its model number is EN-GJS-400-18. The specific ingredients of the material are shown in Table 1. Ductile iron casting products have the characteristics of higher strength, toughness, wear resistance, Etc. By spheroidizating and inoculating, the mechanical properties of ductile iron have been effectively improved.

Tab1 EN-GJS-400-18 chemical composition

\begin{tabular}{ccccccccccc}
\hline element & $\mathrm{C}$ & $\mathrm{Si}$ & $\mathrm{Mn}$ & $\mathrm{P}$ & $\mathrm{S}$ & $\mathrm{Cr}$ & $\mathrm{Ni}$ & $\mathrm{Cu}$ & $\mathrm{Mg}$ & others \\
\hline $\begin{array}{c}\text { content } \\
/ \mathrm{wt} \%\end{array}$ & 2.63 & 3.43 & 0.182 & 0.547 & 0.0043 & 2.93 & 4.46 & 0.062 & 0.137 & $/$ \\
\hline
\end{tabular}

\section{Casting Structure Analysis}

The outline of the case is $1550 \times 540 \times 960 \mathrm{~mm}$. Since the casting structure size is large, there will be a risk that the molten metal may not fill the mold cavity completely during casting. The bottom 
of the lower case is thick, therefore a hot knot is easily formed at the corner which may lead to shrinkage and other defects [2]. In consideration of the complicated shape of the casting and the maximum cross section that is not at the end of the casting, two case molding way is adopted.

\section{Design of casting process}

\section{Pouring position of the casting}

The pouring position of the casting means its position in the mould. It can affect the solidification sequence and the quality of casting [3]. Pouring position of the casting is chose as showed in the Fig.2.
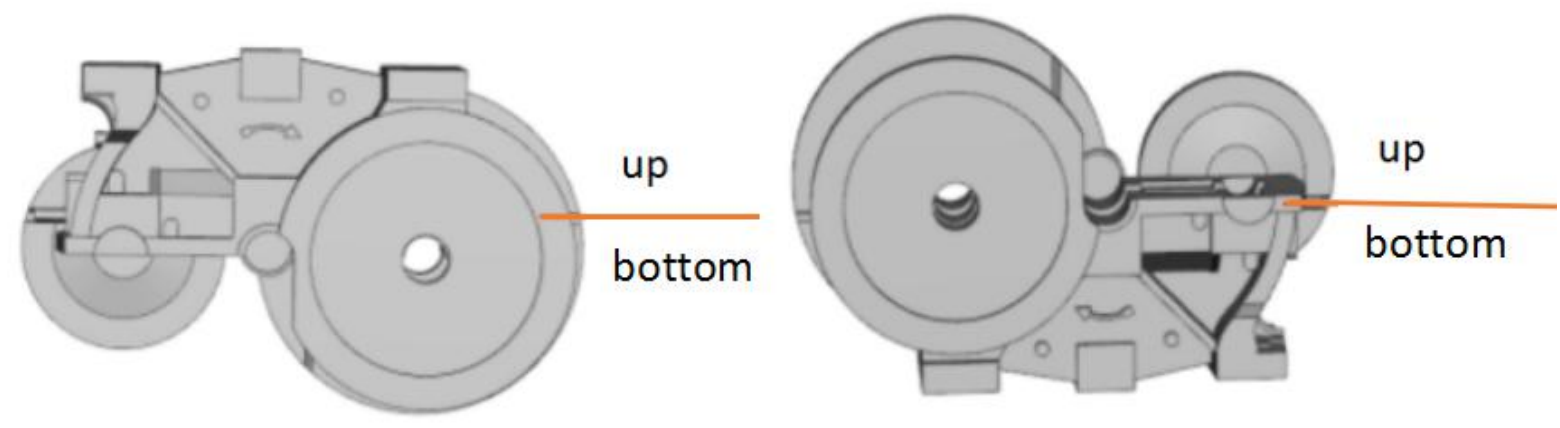

Fig.2 position of the casting

\section{Design of gating system}

The parting surface is selected in the middle of the casting by two-part moulding. The case is located between the upper and lower sandbox and its height is $1212 \mathrm{~mm}$. The sizes of the gate, sprue and runner are calculated respectively, and the sprue shape is determined corresponding to them.
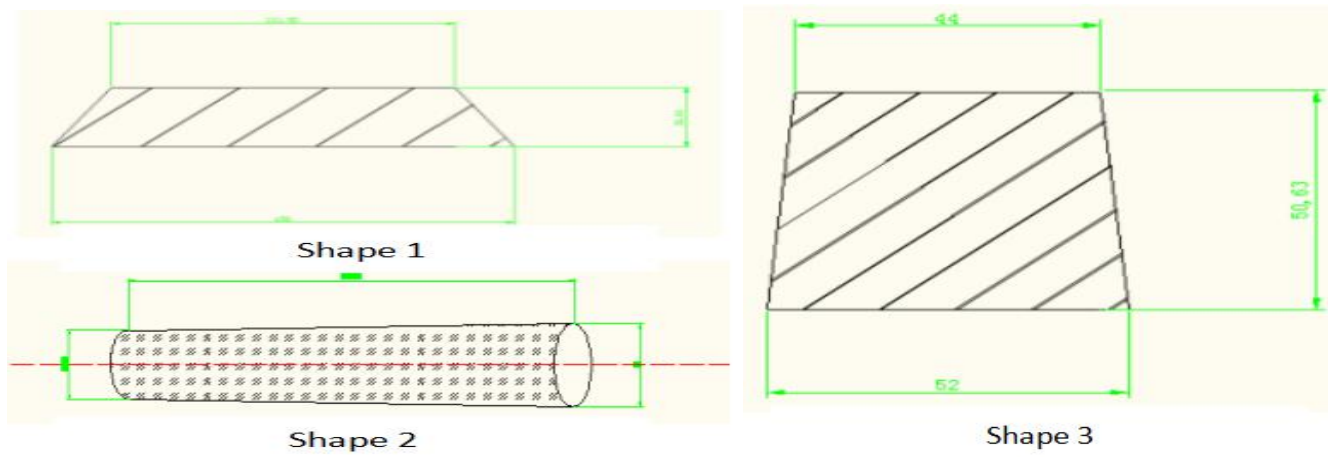

Fig. 3 shape of the gates

As shown in Fig. 3, the size of gate is $47.76 \mathrm{~cm}^{2}$ in shape 1. The size of sprue is $95.32 \mathrm{~cm}^{2}$ in shape 2 and the size of runner is $28.82 \mathrm{~cm}^{2}$ in shape 3 . A sprue base is designed at the turning of the sprue to avoid sand shakeout which is caused by molten metal falling from the sprue.

\section{Design of risers and cold iron}

In order to obtain high-quality castings, not only a reasonable casting system, but also the appropriate feeding capacity is required. Nodular cast iron is characterized by "mushy solidification". There will be precipitation of carbides during solidification. The expansion of the precipitated carbons can reduce the total amount of the required supplemental metal. This feature is 
also called the ductile iron self-feeding [4]. For large castings, it is impossible to rely on the self-feeding of the material. Consequently, it is still necessary to add risers to the casting. According to the hot circle method, three risers are designed on the casting.

By the adding of cold irons, local metal liquid is rapidly solidified to improve the surface hardness and wear resistance of the casting. Three cold irons are added to avoid the partially shrinkage cavity.

So the pouring system was designed as shown in the Fig4.

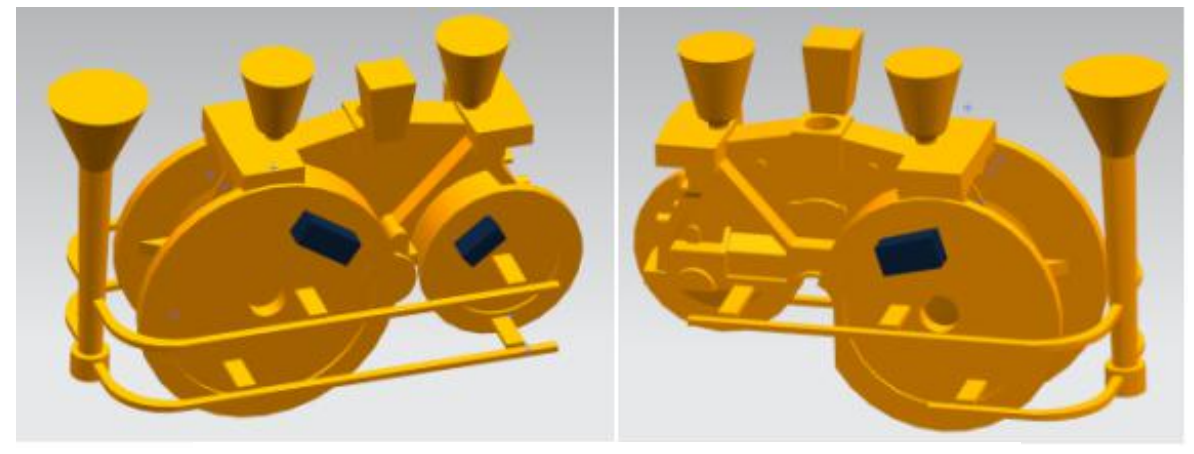

Fig4. pouring system

\section{Design of sand core}

The role of sand core is to form the cavity of the casting. Because of the complex of case shape ,the sand core is designed in the form of coordination with each other. Eleven sand cores are designed to help casting forming. The sand core is placed in the cavity in the sequence of $8 / 9 / 10 / 11 \rightarrow 8 \rightarrow 3 / 4 / 5 \rightarrow 1 / 2 \rightarrow 6 / 7$

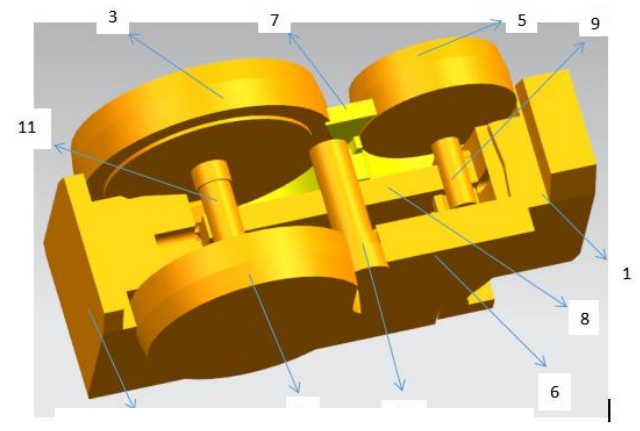

Fig5.sand core assembly

\section{PROcast simulation}

\section{Meshing of the casting}

Firstly, the mesh size of solid sand box is set to $100 \mathrm{~mm}$ and the casting's mesh size is set to 40 $\mathrm{mm}$. In the process of division, some grids are irregular. Automatic repair and manual trimming are used to obtain the desired mesh shape for the casting simulation. The mesh result is shown in Figure 6. 

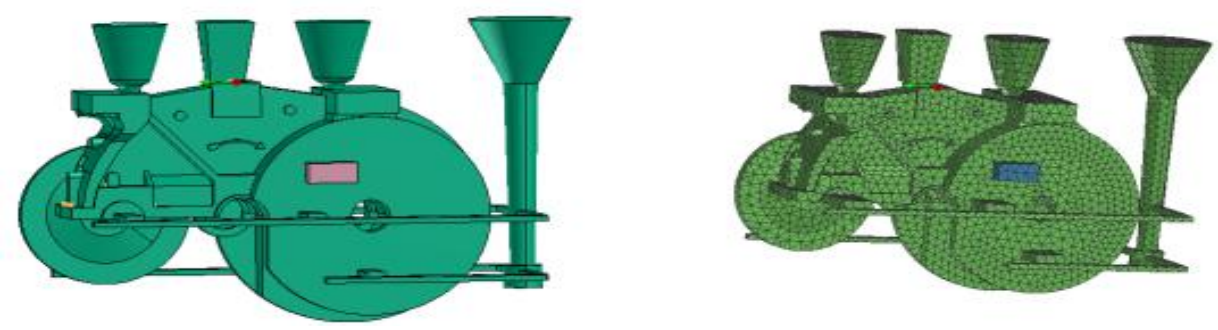

Fig6.the meshing result of the casting

Subsequently, parameters are set up as Tab.2.

Tab.2 Parameters of the casting

\begin{tabular}{lccccccc}
\hline Material & Heat 1 & Heat 2 & $\begin{array}{c}\text { casting } \\
\text { temperature }\end{array}$ & Gravity & $\begin{array}{c}\text { pouring } \\
\text { speed }\end{array}$ & $\begin{array}{c}\text { Casting } \\
\text { type }\end{array}$ & $\begin{array}{c}\text { Initial } \\
\text { temperature }\end{array}$ \\
\hline $\begin{array}{l}\text { Nodular cast } \\
\text { iron }\end{array}$ & $400 \mathrm{~W} / \mathrm{M}^{2} / \mathrm{K}$ & $500 \mathrm{~W} / \mathrm{M}^{2} / \mathrm{K}$ & $1420^{\circ} \mathrm{C}$ & $9.8 \mathrm{~m} / \mathrm{s}^{2}$ & $81 \mathrm{~mm} / \mathrm{s}$ & $\begin{array}{c}\text { Gravity } \\
\text { filing }\end{array}$ & $20^{\circ} \mathrm{C}$
\end{tabular}

\section{Simulating result}

The molten mental is filled to the cavity at the speed of $81 \mathrm{~mm} / \mathrm{s}$. Casting filling process is smooth. During solidification, cold iron plays a very important role in chilling the thick wall [5]. Combined with the final gas and shrinkage porosity diagram, it is found that the concentrated parts of shrinkage cavity and shrinkage porosity have been basically eliminated. As the filling process is more stable, the phenomenon of wrapped gas is basically eliminated, and no turbulence is found. The simulating result can be found in Fig7.

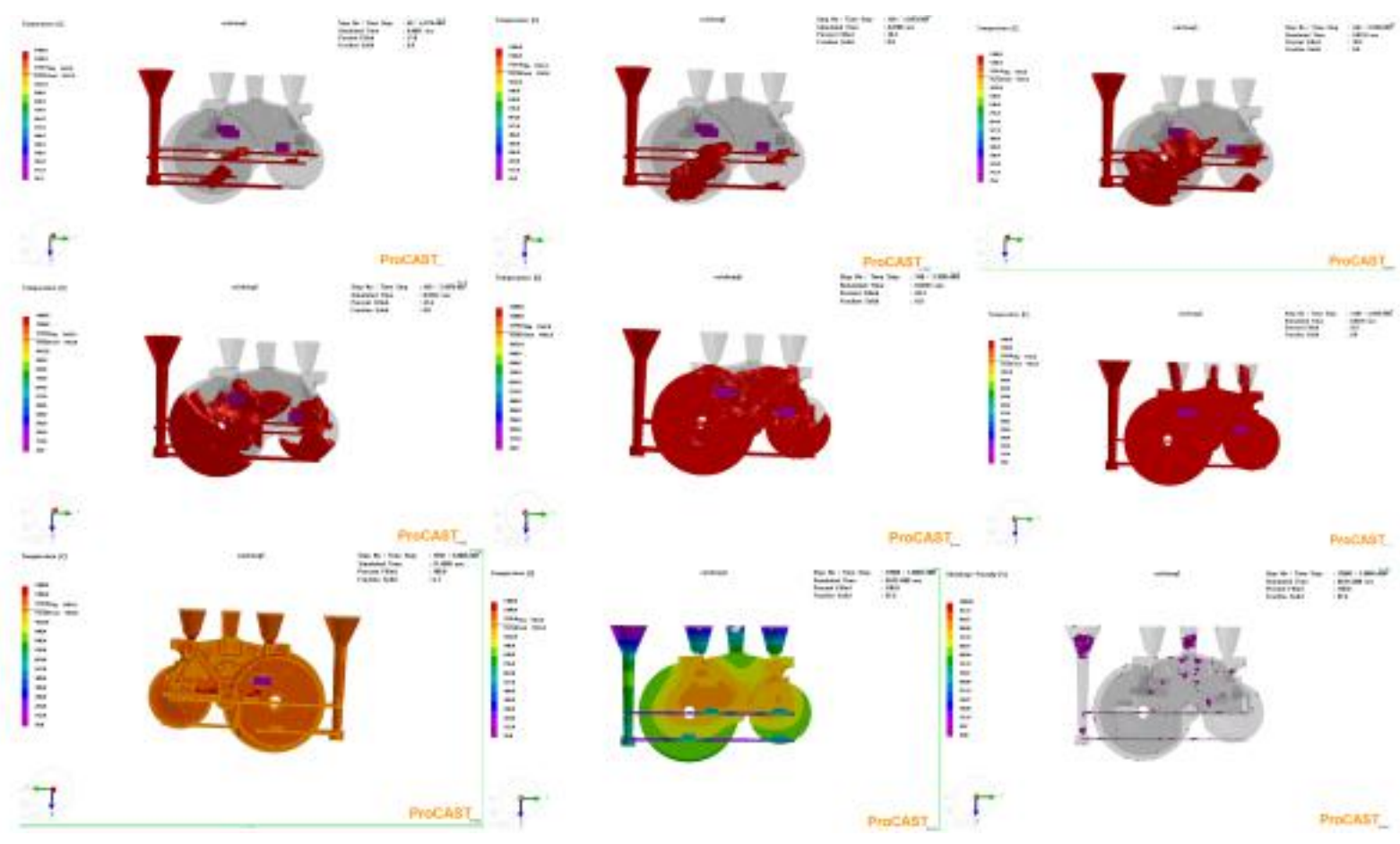

Fig7.the meshing result of the casting 


\section{References}

[1] Qi Chen, Zhaodi Peng, Practical casting manual, M.casting, 08(2009), 27-63

[2] A.Meena, M. El Mansori. Correlative thermal methodology for castability simulation of ductile iron in ADI production[J]. Journal of Materials Processing Technology. 2012, 484-249.

[3] Heisser C, Sturm J C. Casting Process Simulation of Compacted Graphite Iron (03-025). In:Proceeding of the 107th Casting Congress, Milwaukee, Wisconsin, April 26-28, 2003, 685-692.

[4] Radovic Z, Lalovie M. Numerical simulation of steel ingot solidification process[J]. Journal of Material Processing Technology, 2005,16(3) , 156-159.

[5] Svensson, I L, Wessen M. Foundry of cast irons: processing and simulation [C]// Numerical Simulation of Foundry Processes, Sept., 2001 , 87-145. 